\title{
Research and design of efficient resource scheduling model for asymmetric network
}

\author{
Wangjin $^{1}$, Jinchangjiang ${ }^{2}$ \\ Chengdu Aeronautic polytechnic, Chengdu Sichuan 610100, China
}

Keywords: resource scheduling; catfish PSO algorithm; CF-PSO cloud computing

\begin{abstract}
The traditional algorithm used for resource scheduling modeling in asymmetric network, is time-consuming, and has bigger error. The cloud computing resource scheduling method based on catfish PSO algorithm is proposed. By adopting the particle encoding method initialize the particle swarm, and through the fitness function, realize cloud computing resource scheduling effectively based on catfish PSO in asymmetric network. The simulation results prove that, the catfish PSO algorithm for cloud computing resource scheduling in asymmetric network has certain robustness and distributivity, improving work efficiency of scheduling.
\end{abstract}

\section{Introduction}

In the state of speed limited local asymmetric network, the network source scheduling is an important standard to consider the level of performance [1, 2]. At present, MAX-MIN algorithm is mainly used for network source scheduling [3], in this method, each resource needed to scheduling are seen as a scheduling node, to make the user machine iteration from each node and gather resources. If it needs to complete the massive resource scheduling, the calculation load will increase user machine's load [4-5].

Aiming at these defects, this paper presents a cloud computing resource scheduling method based on catfish PSO algorithm. By adopting the particle encoding method initialize the particle swarm, through the fitness function, realize cloud computing resource scheduling effectively based on catfish PSO in asymmetric network, accelerating the rapid of the algorithm, but also to speed up the ability of searching the optimal solution. The simulation results prove that, the cloud computing resource scheduling method based on catfish PSO algorithm has good convergence speed and stability, and saves a large amount of computing resources, better improving work efficiency of scheduling.

\section{Principle of resource scheduling in asymmetric network}

In asymmetric networks, when all kinds of resources linked into a space, it is easy to appear the problem of unbalanced resources distribution. In order to solve such problems, it needs for resource scheduling based on various parameters. Because the changing time of each task in their respective resources is influenced by many external reasons, it is not static, how to solve this problem effectively is a key of reasonable resource scheduling. The resource scheduling in asymmetric network can be described as below:

Assuming that the total number of resource scheduling tasks in asymmetric network is: $n$, the total number of resources in system is: $m . J=\left(J_{1}, J_{2}, \ldots J_{n}\right)$ represents: $n$ single user tasks, $i$ : the need for cost, $m$ resources are taken as a distribution space, task scheduling is to divide $n$ independent tasks into $m$ resources to solute whole space. As the above details, it can get a task scheduling matched the requirements of service quality, as shown in Figure 1: if $|J|=n,|R|=m$, figure 1 is that task $i$ is the cost in the resource 1 to finish solving, it can be expressed as: $1 \leq i \leq n, 1 \leq i \leq m$ i.e. the task allocation problem, could be described by formula (1):

$$
f=\operatorname{Min} \sum_{j \in R} e \cdot j
$$

Represented resource $i$ is the edge of the end, $j$ is the task which can get the resources $i$, if a new node is generated in the system, resource nodes in Figure 1 are relative increased, if the node in the 
network leaves, the resource node would return to the failure information to the task skip device, and delete from the resource set.

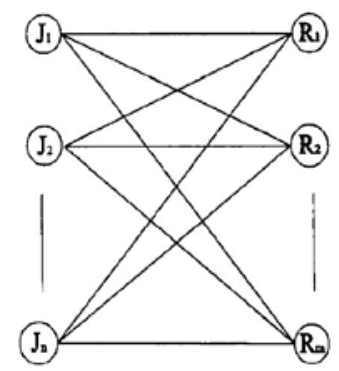

Figure 1 task allocation map

Using the traditional algorithm for resource scheduling in the state of LAN speed limit, resource scheduling strategy is that put each resource machine as a node, let user iterative induct parameters from each node, so resource information in the transformation cannot be updated in a timely manner. If it needs for massive resources, it will cause the load to the equalizer and node, there is no correctness guarantee. Therefore in the state of LAN speed limit, to establish a reasonable and effective resource scheduling can play a great help for improving the efficiency of the work.

\section{Cloud computing resource scheduling algorithm based on catfish PSO algorithm}

Catfish PSO algorithm. In the standard PSO algorithm, the potential solutions of problem needed to optimize can be considered as a point in n-dimensional search space, called the "particle". Particles fly with a certain speed in the search space, the speed is made dynamic adjustment based on their own and companion flying experience, the advantage and disadvantage extent of particle is decided by fitness value [11]. The optimal position of particles and whole groups are set as pbest and gbest, the particle velocity and position update as:

$$
\begin{gathered}
v_{i, d}^{k+1}=\omega \cdot v_{i, d}^{k}+c_{1} \cdot \operatorname{rand}() \cdot\left(\text { pbest }_{i, d}^{k}-x_{i, d}^{k}\right)+ \\
c_{2} \cdot \operatorname{rand}() \cdot\left(\operatorname{gbest}_{d}^{k}-x_{i, d}^{k}\right) \\
x_{i, d}^{k+1}=x_{i, d}^{k}+v_{i, d}^{k+1}
\end{gathered}
$$

In the formula, $\omega$ is the inertia weight; $c_{1}$ and $c_{2}$ are the learning factor; rand() is random number between $(0,1) ; v_{i, d}^{k}$ and $x_{i, d}^{k}$ are the speed and position of particle $i$ in the $d$-dimension in the $k$ iterations respectively; pbest $t_{i, d}^{k}$ is the individual extremal position of particle $i$ in the $d$-dimension; gbest $_{d}^{k}$ is the global extremal position of the group in the $d$-dimension.

\section{Design of Resource scheduling algorithm under cloud computing.}

1)Particle encoding method

Assuming that it has TASK tasks and RESOURCE resources, each task is divided into several sub tasks (subTask) and the total number of subtasks SUBTASK is greater than the number of resources RESOURCE, then the SUBTASK calculation formula is:

$$
\text { SUBTASK }=\sum_{t=1}^{\text {TASK }} \operatorname{taskNum}(t)
$$

In the formula, taskNum ( $\mathrm{t}$ ) is the number of sub task which is contained in the $\mathrm{t}$-th task. $\mathrm{m}[\mathrm{i}, \mathrm{j}]$ represents the serial number of the $\mathrm{j}$-th sub tasks in the $\mathrm{i}$-th task, the encoding method for $\mathrm{m}[\mathrm{i}, \mathrm{j}]$ is :

$$
m[i, j]=\sum_{k=1}^{i-1} \operatorname{taskNum}(k)+j
$$

When TASK=3, RESOURCE=3, SUBTASK=10, particle $(3,2,1,3,2,1,1,3,2,1)$ represents a potential scheduling strategy, the encoding mode is in table 1 . Task, sub task $(2,3)$ represents that the number of first sub task is 3 in second task; sub tasks, resources pair $(1,3)$ represents that task 1 is allocated to resources 3 to handle.

2)To initialize the particle swarm

$\mathrm{S}$ particles are randomly generated, the $\mathrm{i}$-th particle position is represented by a vector $\mathrm{xi}$, $\mathrm{xi}$ is defined as $x i=\{x i 1, x i 2, \ldots, x i n\},(1 \leq n \leq S U B T A S K, \quad 1 \leq i \leq S)$, xij represents that the task $j$ is assigned 
to the resources $x i$. Speed is represented by vi vector, defining $v i=\{v i 1, v i 2, \ldots, v i n\}$, RESOURCE $\leq \mathrm{vij} \leq \mathrm{RESOURCE}$. the position of initialized particle is between [1,RESOURCE], , the speed of the particle is taken randomly between [- (RESOURCE-1), (RESOURCE-1)].

3) The fitness function

Let the number of resources in the cloud system for RESOURCE, resource(r,i) is the time to perform the i-th sub task of $r$-th resource, with article $s$ of the resources of, $\beta(r)$ is the resource level of $\mathrm{r}$-th resource, $\mathrm{n}$ is the number of sub tasks assigned to the resources, the calculation formula for the total time to complete all the tasks is :

$$
R S F T=\max _{r=1}^{R E S O R C E} \beta(r) \times \sum_{i=1}^{n} \text { resource }(r, i)
$$

The particle fitness function is defined as the reciprocal of the task complete time:

$$
F(i)=\frac{1}{R S F T(i)}
$$

Cloud computing resource scheduling steps based on CF-PSO.

(1) according to the cloud computing system resources and the number of tasks, initialize the particle swarm.

(2) according to equation (10) , calculate the fitness function value of particle, and choose the optimal particle as the individual optimal solution(pbest) and the group optimal solution (gbest).

(3) according to the formula (5), (6) determine the catfish operator, and update the particle velocity and position.

(4) the fitness value of each particle in the new position is calculated, if the particle's fitness value is better than that of pbest $_{i}$, pbest is $_{i}$ updated to the new location.

(5) judge the termination condition, if satisfied, the global optimal particle position is decoded, to get the optimal cloud computing resources scheduling scheme, otherwise, to continue the iteration.

The cloud computing resource scheduling process based on CF-PSO algorithm is shown in Figure 1.

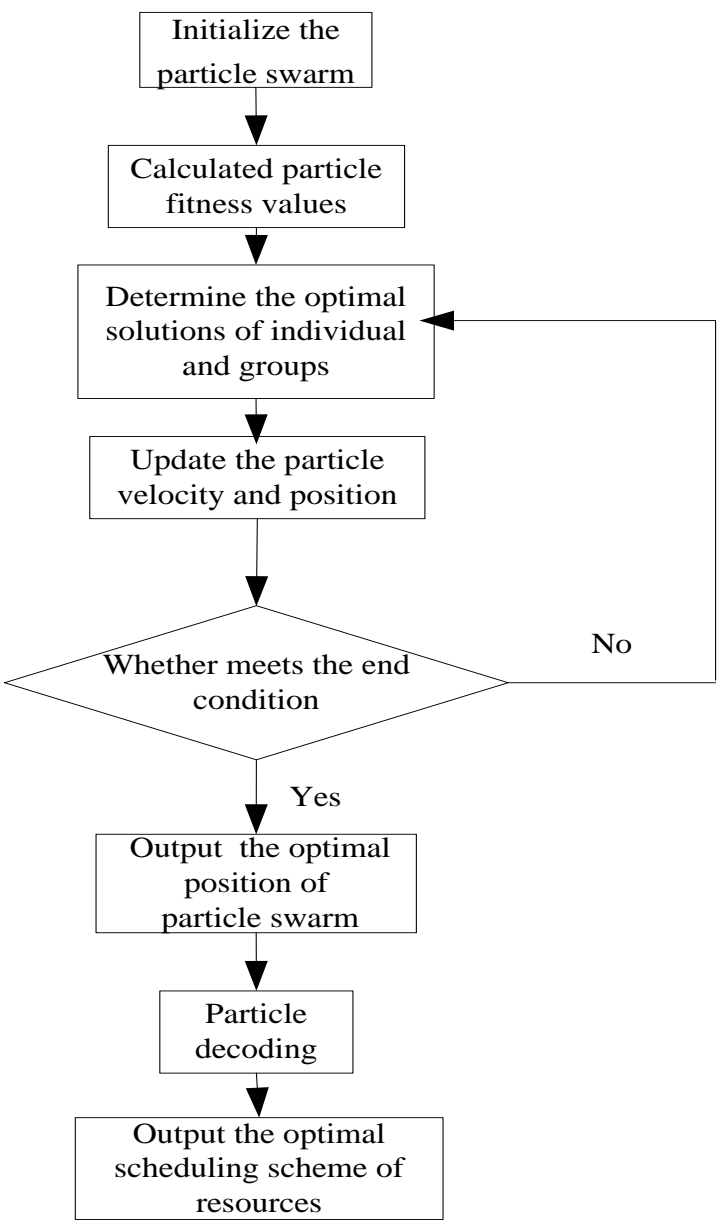

Figure 1 cloud computing resource scheduling process of CF-PSO 


\section{Experiments and results analysis}

In order to prove that real effectiveness of the proposed cloud computing resource scheduling method based on the catfish PSO algorithm, it needs an experiment. The first model for resource scheduling of asymmetric network is established. As follows:

This experiment finds 7 operations, 2 resource nodes randomly, if each node has the same bandwidth, and the task set of each node is 0 , a simulation experiment is carried out using the proposed algorithm and the hybrid ant colony algorithm, the main parameters are $p_{\circ}=0.6, p=0.8, \mu_{0}=0.9, b=0.8, \alpha=3, \beta=4$, other experimental data showed in table 1 :

Table 1 time-table of resource scheduling task

\begin{tabular}{lll}
\hline & R1 & R2 \\
\hline J1 & 4 & 8 \\
J2 & 4.5 & 9 \\
J3 & 1.5 & 3 \\
J4 & 2.5 & 5 \\
J5 & 5 & 10 \\
J6 & 2.25 & 4.5 \\
J7 & 9 & 3 \\
\hline
\end{tabular}

Table 2 task allocating table

\begin{tabular}{|l|l|l|}
\hline & R1 R2 & The optimal results \\
\hline MAX-MIN & J5J6, J1J7 & 11.0 \\
\hline HAC & J2J6, J3J7 & 7.0 \\
\hline
\end{tabular}

The data in Table 1 is set as the experimental data. Using traditional algorithm and the proposed algorithm respectively for scheduling and finally to get the optimal value of 7.0.

it can be obtained from Table 2, the distribution results by this algorithm are: $R 1=\{J 2, J 6\}, R 2=\{J 3, J 7\}$, the optimal value is 7.0, the value of distribution indicates that the proposed cloud computing resource scheduling method based on catfish PSO algorithm have better results than traditional algorithm.

The proposed algorithm and GA, PSO algorithm are respectively used for experiments to get the accuracy of different algorithms showed in Figure 2

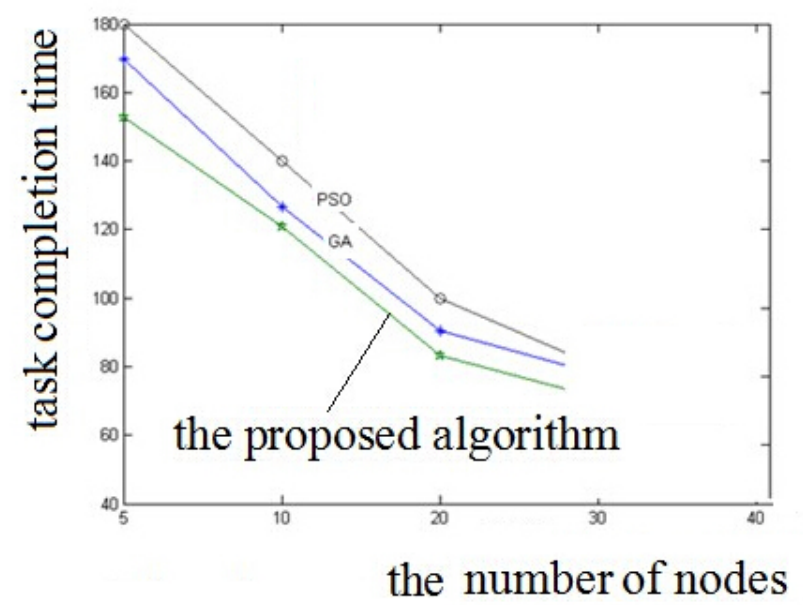

Figure 2 the relationship between task completion time and the number of nodes changes According to figure 2, table 3 can be obtained by analysis 
Table 3 Comparison of the accuracy of different algorithms

\begin{tabular}{lllll}
\hline The number of experiments & $\begin{array}{l}\text { Efficiency of } \\
\text { algorithm\% }\end{array}$ & $\begin{array}{l}\text { proposed } \\
\text { algorithm\% }\end{array}$ & traditional \\
\hline 1 & 98 & 85 & \\
2 & 97 & 82 & \\
3 & 95 & 80 & \\
4 & 96 & 83 & \\
5 & 94 & 79 & \\
6 & 97 & & 82 & \\
\hline
\end{tabular}

On the basis of the above experiments it can be learned, that using the method of catfish particle swarm algorithm for resource scheduling in asymmetric network state is very effective, it can ease the burden of computer users, and improve work efficiency, to meet the needs of the reasonable resources scheduling in asymmetric network.

\section{Conclusions}

Through the cloud computing resource scheduling method based on catfish PSO algorithm, by adopting the particle encoding method initialize the particle swarm, and through the fitness function, realize cloud computing resource scheduling effectively based on catfish PSO in asymmetric network. The simulation results prove that, the catfish PSO algorithm for cloud computing resource scheduling in asymmetric network has certain robustness and distributivity, improving work efficiency of scheduling.

\section{References}

[1] Weng Chuliang, Lu Xinda. A grid resource pricing algorithm based on market mechanism [J]. Journal of computer research and development, 2004, 41 (7): 1151-1156).

[2] Zhang Han, Wang Xiufeng, Li Zhaohui, et al. The HASH function based on spatiotemporal chaos system construction [J]. Acta physica Sinica, 2005, 54 (9): 4006-4011.

[3] Liu Guangjie, Shan Liang, Sun Jinsheng, et al. The HASH function based on spatiotemporal chaos system construction [J]. Control and decision, 2006, 21 (11): 1244-1248.

[4] Zhao Geng, Yuan Yang, Wang Bing. One-way HASH function construction based on Cross coupled map lattice [J]. Journal of Southeast University (NATURAL SCIENCE EDITION), 2009, 39 (4): 728-732.

[5] Zhang Jianxun, Gu Zhimin, Deng Chao. Review of the advances in study on cloud computing [J]. Computer application research, 2010, 27 (2): 429-433

[6] Yang Shengwen, mei-lin shi. A supporting QoS constraints of Web services discovery model [J]. Journal of computers, 2005, 28 (4) : 589-594.

[7] Zhang Lifang. P2P large database data files based on genetic algorithm scheduling policy research [J]. Computer and digital engineering, 2009, 5 (4) : 31-33.

[8] Shao-hua qin, zi-mu li qing-song CAI, et al. A large database based on proxy cache data files dynamic scheduling algorithm research [J]. Journal of computers, 2005, 28 (2) : 185-194. 